\section{Arbeitgeber muss Ex-Mitarbeiter aus Web löschen}

Arztpraxen, Kliniken und andere Arbeitgeber müssen Daten ausgeschiedener Arbeitnehmer von ihrer Homepage löschen. So entschied das Hessische Landesarbeitsgericht die Klage einer Anwältin (19 SaGa 1480/11). Ihr Eintritt in eine Kanzlei wurde mit Foto auf der Homepage sowie in einem News Blog bekannt gegeben. Als sie nach drei Monaten wieder ausschied, löschte die Kanzlei die Daten von der Homepage, nicht aber aus ihrem News Blog. Das Landesarbeitsgericht entschied, dass ausgeschiedene Arbeitnehmer die Löschung ihrer Daten von allen Internetauftritten verlangen können. Martin Wortmann

\section{Rückstellung nur mit Regressbescheid}

Ärzte dürfen erst dann steuerlich wirksame Rückstellungen für Regressforderungen bilden, wenn die Prüfgremien einen Regressbescheid erlassen haben. Das hat das Finanzgericht Bremen entschieden (1 K 32/10 [5]). Stützen sich die Rückstellungen nur auf ein eingeleitetes Prüfverfahren, sind sie steuerlich unwirksam. Im verhandelten Fall wurde gegen eine Gemeinschaftspraxis ein Prüfverfahren eingeleitet, weil sie die maßgeblichen Richtgrößen für die Verordnung von Arznei- und Heilmitteln erheblich überschritten hatte. Die Ärzte hatten in ihren Bilanzen deshalb gewinnmindernde Rückstellungen wegen der befürchteten Festsetzung von Regressen gebildet. Zu Unrecht, so das Gericht.

Rebekka Höhl

\section{Vermittlungsprovision unzulässig}

Für die Vermittlung von Patienten an eine Klinik dürfen Ärzte generell keine Provisionen nehmen. Das gilt selbst für Privatpatienten aus dem Ausland, heißt es in einem Urteil des Landgerichts Kiel (8 O 28/11). Es verwarf damit den Provisionsvertrag zwischen einem Arzt und dem Uniklinikum Schleswig-Holstein als sittenwidrig. Der Arzt spricht fließend Arabisch und verfügt über gute Kontakte in den arabischen Raum. Der Arzt sollte laut Vertrag ausländische Privatpatienten vermitteln und sie anschließend während des Klinikaufenthalts begleiten und dolmetschen.

Martin Wortmann

\section{Praxischefs müssen seit April mehr Gehalt zahlen}

\author{
Ein Plus von 2,9\% für Medizinische Fachangestellte (MFA) und von rund \\ $8 \%$ für Auszubildende - so sieht der neue MFA-Tarif aus.
}

$\mathrm{S}_{\mathrm{N}}^{\mathrm{e}}$ eit April steigen die Tarifgehälter für Medizinische Fachangestellte (MFA). Anders als bei der Tarifrunde in 2011, als das prozentuale Plus für Berufseinsteiger wird es dieses Mal für alle MFA ein und dasselbe Lohnplus geben, nämlich 2,9\% auf das bisherige Tarifgehalt. Darauf haben sich der Verband der medizinischen Fachberufe $(\mathrm{VmF})$ und die Arbeitsgemeinschaft zur Regelung der Arbeitsbedingungen von Medizinischen Fachangestellten/Arzthelferinnen (AAA) geeinigt.

\section{Auszubildende bekommen noch mehr}

Ein dickeres Gehaltsplus gibt es für die Auszubildenden: Sie erhalten prozentual gesehen ein Plus zwischen $7 \%$ und $8 \%$ : höher lag als das für erfahrene Kräfte,
Das Gehalt für das erste Ausbildungsjahr steigt von $561 €$ auf nun $610 €$, für das zweite Ausbildungsjahr von $602 €$ auf 650 $€$ und für das dritte Ausbildungsjahr von $646 €$ auf $700 €$.

Sein Ziel, für jede MFA mindestens einen Stundenlohn von 10 Euro auszuhandeln, habe der VmF mit dem neuen Abschluss leider noch nicht erreicht, sagt Margret Urban, zuständig für das Ressort Tarifpolitik beim Verband. Man liege bei den Berufseinsteigern durch den neuen Tarif bei einem Stundenlohn von 9,21 €. „Wir hätten schon gerne eine drei vor dem Komma gehabt", so Urban. Die Vorsitzende der AAA, Dr. Cornelia Goesmann, hingegen sieht in den 2,9\% die Grenze dessen, was für die Ärzteschaft möglich war.

Rebekka Höhl

\section{Abrechnungstipp!}

\section{Telefonische Beratung - nicht immer nur die Nr. 1 GOÄ}

— Welche Leistungen sind außer der Nr. 1 in der GOÄ auch bei telefonischer Erbringung abrechnungsfähig? Ohne jeden Zweifel, weil im Legendentext so formuliert, ist die Nr. 3, die eingehende, das gewöhnliche Maß übersteigende Beratung ebenfalls per Telefon zu erbringen und abzurechnen. Die ersten Zweifel kommen schon bei der Nr. 4, Erhebung der Fremdanamnese eines Kranken und/oder Unterweisung der Bezugsperson(en) - im Zusammenhang mit der Behandlung eines Kranken. Beide Teilleistungen sind Leistungen, die ohne Qualitätsverlust auch per Telefon zu erbringen sind. Da in der GOÄ an keiner Stelle die telefonische Leistungserbringung für diese Position ausgeschlossen ist, dürfte eine Abrechnung möglich sein. Dies sollte aber nicht regelhaft erfolgen und bei Nachfragen sollte der einzelne Fall erklärbar nachvollziehbar sein, weshalb die telefonische Leistungserbringung erforderlich und auch effektiv war.

Ähnliche Zurückhaltung ist gegeben bei speziellen psychiatrischen Leistungen, den Positionen 806, die „psychiatrische Behandlung durch gezielte Exploration und eingehendes therapeutisches Gespräch, auch in akuter Konfliktsituation“ mit einer Mindestdauer von 20 Minuten sowie die Nr. 812, die „Psychiatrische Notfallbehandlung bei Suizidversuch und anderer psychischer Dekompensation durch sofortige Intervention". In beiden Fällen ist die telefonische Erbringung erlaubt, aber sicher nur in Ausnahmefällen nachvollziehbar erforderlich.

Fazit: Die telefonische Erbringung von Gesprächsleistungen ist keine für die Nr. 1 reservierte Möglichkeit, sondern - bei gezieltem Einsatz - auch bei anderen Gesprächsleistungen möglich.

Dr. med. Heiner Pasch 